\title{
Effects of nasal CPAP on simulated driving performance in patients with obstructive sleep apnoea
}

\author{
C F P George, A C Boudreau, A Smiley
}

\begin{abstract}
Background - Many patients with obstructive sleep apnoea (OSA) have difficulty in driving and experience increased automobile accidents. It has previously been shown that patients with OSA perform poorly on a laboratory based divided attention driving test (DADT).

Methods - Seventeen men with OSA of mean (SD) age 49.7 (11.2) years and an initial apnoea/hypopnoea index (AHI) of 73.0 (28.9) were restudied from one to 12 (mean (SD) 9.2 (4.2)) months after initiating treatment with nasal continuous positive airway pressure (CPAP) to examine the effects of treatment on DADT performance. Eighteen age and sex matched controls were also retested 8.4 (3.4) months after their initial tests. Following a practice session, all subjects were given the DADT for 20 minutes before each daytime nap of the standard multiple sleep latency test (MSLT).
\end{abstract}

Results - Untreated patients with OSA, who performed much worse than controls in all measures, improved significantly on all measures of performance, particularly in tracking error which returned to the level of controls in all but one patient. Changes in performance were much greater for patients with OSA than for controls in tracking error (mean difference $106(95 \%$ CI 75 to 135$) \mathrm{cm})$, sleep latencyl MSLT (5.3 (95\% CI 2.7 to 8.0) $\mathrm{min}$ ), number of correct responses (1.2 (95\% CI 0.4 to 1.9$)$ ), number of missed responses (1.7 (95\% CI 0.9 to 2.3$)$ ), and number out of bounds (10.0 (95\% CI 7.9 to 13.6$)$ ), but not for response time $(0.1(95 \%$ CI -0.3 to $0.2) \mathrm{s})$. Improvement in tracking error was highly correlated with improvement in sleepiness $(r=0.65)$.

Conclusions - Impairment in laboratory driving performance skills in patients with OSA is reversed by successful treatment with nasal CPAP. Changes in daytime sleepiness account for some but not all of the improvement.

(Thorax 1997;52:648-653)

Keywords: sleep apnoea, driving, automobile accidents, performance.

As a group, patients with obstructive sleep apnoea (OSA) have increased automobile accidents when compared with control groups or population data. ${ }^{1-4}$ Increasingly, physicians are being asked to make recommendations about a patient's ability to drive and, in some jurisdictions, are required by law to report sleepy patients. While the recent ATS statement on sleep apnoea and driving makes recommendations on driving, ${ }^{5}$ it would be helpful to have a safe, reliable and valid laboratory based test of driving performance to aid in the process. Recently we have been using a laboratory based divided attention driving task (DADT) ${ }^{6}$ which is sensitive to impairment due to alcohol. When given to a group of patients with OSA we found that at least half of the group performed as poorly, if not worse, than controls impaired by alcohol. Since most patients report improvement or even resolution of their OSA symptoms with treatment, we wondered whether continuous positive airway pressure (CPAP) might improve and/or normalise DADT performance and sleepiness.

\section{Methods}

SUBJECTS

Control subjects and patients with OSA from a previous protocol ${ }^{6}$ made up the study population. Entry criteria were as previously reported, the patient group comprising subjects presenting with snoring and/or daytime sleepiness to the London Health Sciences Centre Sleep Disorders Clinic for evaluation of possible sleep apnoea. Following history and physical examination, those patients suspected clinically of having sleep apnoea were invited to participate in the study. In an attempt to minimise selection bias, consecutive new patients were invited to participate. Exclusion criteria were: (a) no driver's licence (currently, or in the past); (b) presence of physical disability (muscle weakness) which could independently affect driving performance; (c) use of hypnotics/sedatives which could produce sleepiness and/or impair performance; d) use of stimulants which could decrease sleepiness; (e) diagnosis other than OSA; (f) clinical or laboratory evidence of hypothyroidism; and ( $\mathrm{g}$ ) apnoea/hypopnoea index of less than $15 .{ }^{7}$ Subjective sleepiness was neither a necessary nor a sufficient condition for entry to the study. At the time the first study began, the work of Young et $a l^{8}$ had not been published and it was assumed that the prevalence of sleep apnoea was much lower in women ${ }^{9}$ so women were not included.

Age matched control subjects were recruited from the community by advertisement. To reduce the possibility of differences in driving 


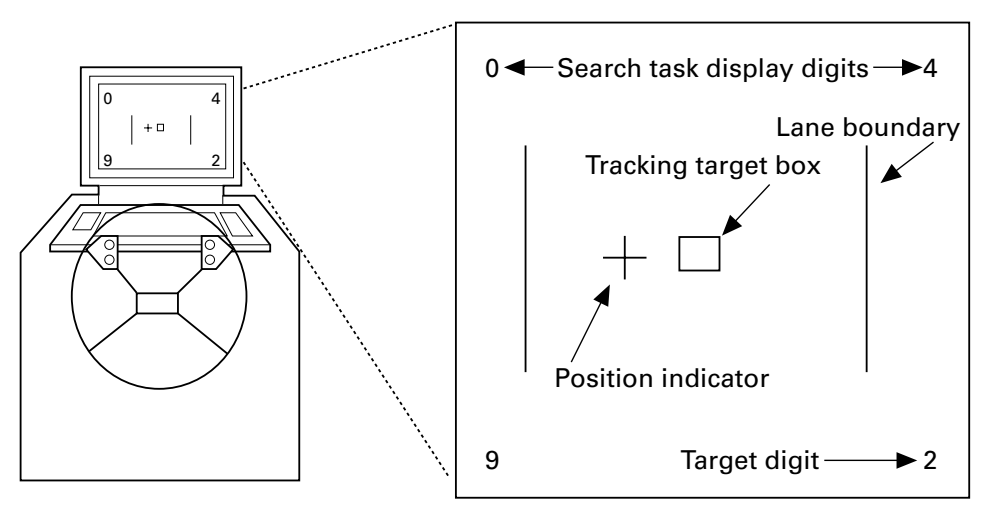

Figure 1 Diagrammatic representation of the driving simulation tests. (A) Steering wheel with mounted buttons interfaced to the computer. (B) Screen display with the elements of the divided attention driving test (see text for details). lane position) - also referred to as lane position variability - and response time to targets. Other measures include the number of correct, incorrect and missed targets, and the number of times the vehicle is out of bounds.

\section{Polysomnography}

Standard overnight polysomnography included electroencephalographic (EEG) data (C1/A2, C4/A1, O2/A1), electro-oculographic (EOG) data and submental electromyographic (EMG) data, bilateral tibialis anterior EMG, expired $\mathrm{CO}_{2}$ (qualitative) or thermocouple at nose and mouth to index airflow, respiration (rib cage and abdominal respiratory inductive plethysmography using Respitrace), and oxygen saturation with ear oximetry (Ohmeda 3700).

related skills between patients and controls we attempted to recruit controls with similar years of driving experience as the patients, especially in terms of current driving exposure. Only healthy subjects who were not using CNS active drugs and who had no complaints of sleepiness were included. Any asymptomatic subject with an apnoea/hypopnoea index of less than 15 was maintained in the control population. ${ }^{7}$

There were 21 patients with OSA, all of whom were treated with nasal CPAP, and 17 were available for retesting 1-12 months after institution of CPAP therapy. Compliance with CPAP was assessed by direct questioning. Eighteen of the initial control population were also restudied 2-12 months later to evaluate test-retest reliability and to determine whether they showed learning effects if testing was repeated. The data set therefore consists of four groups: OSA, OSA-CPAP, control 1, and control 2.

\section{ASSESSMENT PROCEDURE}

Divided attention driving task (DADT)

The details of the divided attention driving task have been previously described ${ }^{6}$ In brief, the primary task involves tracking while the secondary task involves visual search of the periphery. Figure 1 displays the essential features of the test set-up. The cursor is controlled by the steering wheel and the dynamics are those of a vehicle travelling at a constant speed. The cursor is made unstable by a laterally applied random force meant to stimulate randomly appearing side wind gusts and/or changes in the pitch or surface of the road. Subjects attempt to centre the cursor (vehicle) in the centre of the box (road) by using the steering wheel to correct for lateral deviations. Attention is divided between tracking and visual search of the corners of the screen to monitor for a target number. Single digits are displayed in each of the four corners of the screen and change in a random way. Subjects are instructed to push the wheel mounted button corresponding to the corner of the screen in which the target number appears. The main outcome variables from this test are tracking error (standard deviation of
Multiple sleep latency testing (MSLT)

Four naps were carried out and sleep onset was scored from three consecutive epochs of stage 1 sleep or one epoch of any other stage. ${ }^{10}$

\section{Protocol for assessments} somnography followed by repeated DADT. Each performance test was followed by 20 minute naps as part of the MSLT. The time in bed prior to daytime testing was fixed at eight hours regardless of time asleep. Patients with OSA slept with nasal CPAP at the preset pressure used at home and no adjustments were made unless there was obvious apnoea. Control subjects slept at home on the night prior to (repeat) testing and were instructed to have their usual sleep period time. They reported to the laboratory at 08.00 hours.

Following breakfast all subjects (controls and those with OSA) underwent a practice session on the divided attention driving task. As was the case on initial testing, the practice consisted of six five-minute tests. Then, before each of the four naps of the MSLT, a 20 minute divided attention driving test took place and the outcome measures were averaged for the four trials in the day.

\section{STATISTICAL ANALYSIS}

To determine differences during practice runs in the main performance measures, repeated measures analysis of variance was used. Polynomial tests of order were used to test for trends, the first polynomial testing for linear trends. Unpaired $t$ tests were used to compare changes from baseline in patients with OSA to changes from baseline in controls for the main outcome measures of tracking error and response time as well as the secondary measures of correct and missed targets and times out of bounds. Linear regression was used to examine the relationship between change in sleepiness (as measured by MSLT) and performance measures. Paired and unpaired $t$ tests were used to compare ages and measures of sleep
All subjects underwent overnight poly- 
Table 1 Comparison of age, apnoea index and sleep structure in control subjects and patients with obstructive sleep apnoea (OSA), before and after treatment with CPAP

\begin{tabular}{llll}
\hline & Control & OSA & OSA-CPAP \\
\hline Age (years) & $46.1(15.1)$ & $49.3(5.1)$ & $49.7(11.2)$ \\
Apnoea/hypopnoea index & $3.1(6.1)$ & $73.0(28.9)^{*}$ & $6.5(7.1) \dagger$ \\
Awakenings & $27.4(9.2)$ & $32.8(16.7)^{*}$ & $26.2(12.2) \dagger$ \\
Stage changes/hour & $17.3(15.5)$ & $18.4(6.9)$ & $13.7(3.9) \dagger$ \\
Arousals/hour & $9.9(7.7)$ & $67.5(28.5)^{*}$ & $11.4(5.9) \dagger$ \\
Sleep stages (\% TST) & $8.4(4.1)$ & $10.6(6.9)$ & $9.3(6.4)$ \\
$\quad 1$ & $54.3(8.9)$ & $68.3(11.8)$ & $53.1(8.4)$ \\
2 & $18.5(7.2)$ & $6.9(6.8)^{*}$ & $15.0(5.8) \dagger$ \\
SWS (3+4) & $19.0(6.4)$ & $13.2(6.3)^{*}$ & $22.1(6.1) \dagger$ \\
REM &
\end{tabular}

$*$ p $<0.05$ OSA versus control. $\nmid \mathrm{p}<0.05$ OSA-CPAP versus OSA.

fragmentation. Comparison of sleep stages were restricted to slow wave sleep (SWS, stages 3 and 4 combined) and REM sleep.

\section{Results}

SUBJECTS

Seventeen patients with OSA and 18 control subjects were retested. The two groups were similar in age (controls 45.6 (14.6) years, patients with OSA 49.7 (11.2) years) and their ages did not differ from the larger groups from which they were drawn (see above).

POLYSOMNOGRAPHY

The results of the sleep studies for each test for the two groups are shown in table 1. Untreated patients with OSA had considerable sleep fragmentation with significantly increased microarousals per hour of sleep (67.5 (28.5) versus 9.9 (7.7), p <0.001) and awakenings (32.8 (16.7) versus $27.4(9.2), \mathrm{p}<0.05)$ compared with controls. Sleep improved markedly with CPAP treatment and there were no significant
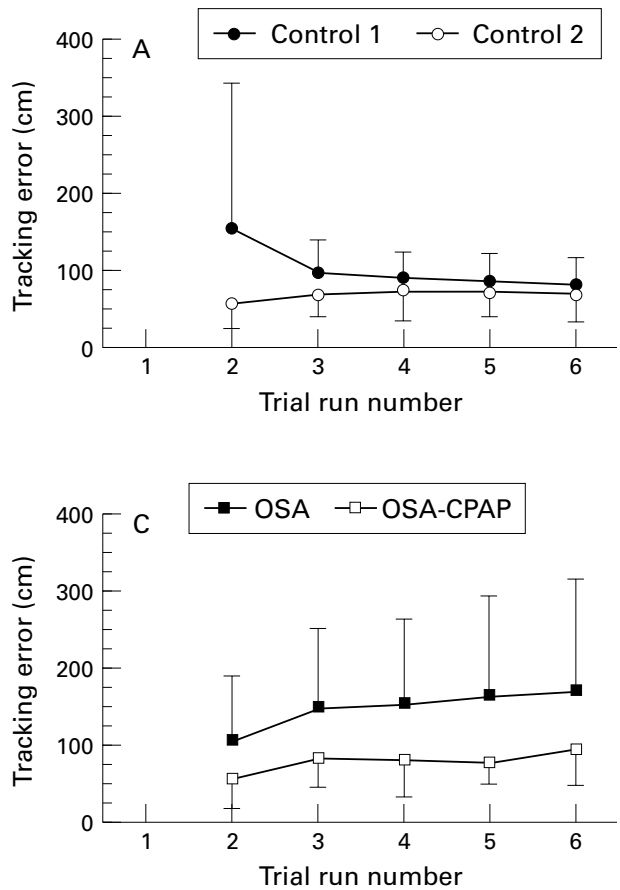

differences in overnight sleep measures between the CPAP treated group and the controls. CPAP sleep studies and repeat divided attention driving tests were performed 9.2 (4.2) months (range 1-12) after initiating treatment and control subjects were retested 8.4 (3.4) months (range 2-12) after their first studies. Reported compliance with CPAP was high with all subjects claiming CPAP use of six or more hours for six or more nights per week.

\section{PERFORMANCE LEARNING EFFECTS}

The results from the practice trials are shown in fig 2. Trial 1 involved visual search alone, trial 2 was tracking alone, and trials 3-6 involved both tasks. For control subjects there were no differences in performance in runs 3-6 (within-test learning) or between practice assessments (control 1 versus control 2; fig 2). The same applied to patients with OSA except that the magnitude of the tracking error was much less in those treated with CPAP than in the untreated patients ( $p<0.001$, fig $2 \mathrm{C}$ ). Regardless of condition, patients with OSA learned at the same rate as the control subjects.

\section{PERFORMANCE: TREATED VERSUS UNTREATED}

OSA

Performance on all measures improved markedly with treatment in patients with OSA (table 2). Changes in performance were much greater for the OSA patients than for controls in tracking error (mean difference (95\% CI) 106 (75 to 135$) \mathrm{cm}$ ), sleep latency/MSLT $(5.3(95 \%$ CI 2.7 to 8.0 ) $\mathrm{min}$ ), number of correct responses (1.2 (95\% CI 0.4 to 1.9$)$ ), number of
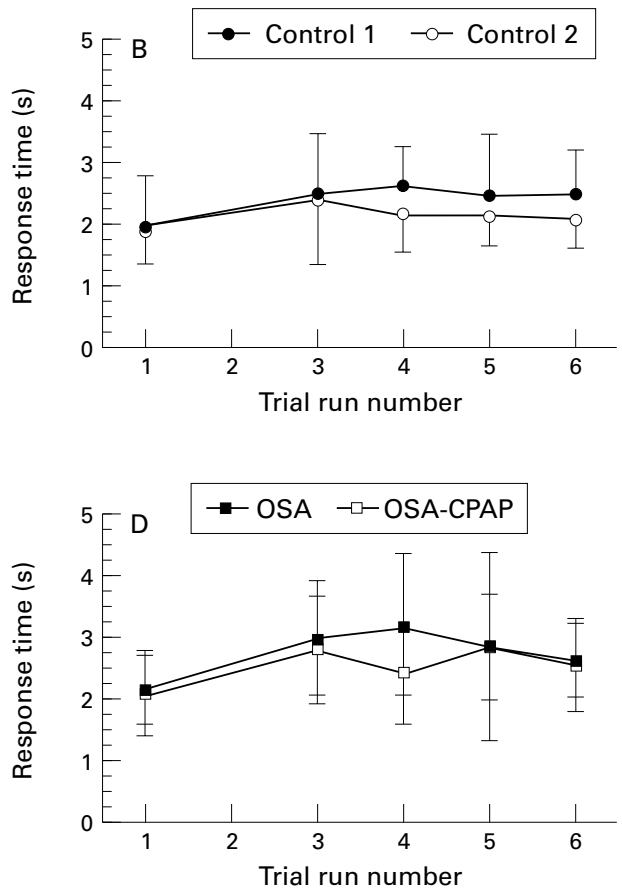

Figure 2 Effect of practice on performance measures - tracking error $(A$ and $C$ ) and response time $(B$ and $D)-i n$ control subjects $(A$ and $B)$ and patients with $O S A(C$ and $D)$. The results are represented as means with one standard deviation. There was no strong learning effect for either measure. 
Table 2 Mean (SE) repeated daytime sleep and performance measures in controls and patients with obstructive sleep apnoea (OSA) before and after treatment with CPAP

\begin{tabular}{|c|c|c|c|c|c|c|c|}
\hline & \multirow{2}{*}{$\begin{array}{l}\text { Control } 1 \\
\text { (C1) }\end{array}$} & \multirow{2}{*}{$\begin{array}{l}\text { Control } 2 \\
\text { (C2) }\end{array}$} & \multirow{2}{*}{$\begin{array}{l}O S A \\
(O S A 1)\end{array}$} & \multirow{2}{*}{$\begin{array}{l}\text { OSA-CPAP } \\
(O S A 2)\end{array}$} & \multicolumn{3}{|l|}{ Difference } \\
\hline & & & & & $C 2-C 1$ & OSA2-OSA1 & $p$ value \\
\hline MSLT (min) & $12.3(0.9)$ & $12.9(0.8)$ & $7.2(0.8)$ & $13.2(0.8)$ & $0.6(0.9)$ & $5.9(1.0)$ & $<0.001$ \\
\hline Tracking error $(\mathrm{cm})$ & $82(4.8)$ & $88(6.9)$ & $228(17.2)$ & $113(9.5)$ & $6(4.9)$ & $115(14.3)$ & $<0.001$ \\
\hline Response time (s) & $2.6(0.1)$ & $2.3(0.1)$ & $3.2(0.1)$ & $2.8(0.1)$ & $-0.3(0.1)$ & $-0.4(0.1)$ & NS \\
\hline Correct responses (n) & $39.3(0.1)$ & $39.7(0.1)$ & $36.2(0.5)$ & $37.8(0.5)$ & $0.4(0.2)$ & $1.6(0.3)$ & $<0.05$ \\
\hline Missed responses (n) & $0.6(0.1)$ & $0.4(0.1)$ & $3.7(0.5)$ & $2.2(0.5)$ & $-0.2(0.1)$ & $1.5(0.4)$ & $<0.05$ \\
\hline Out of bounds (n) & $0.1(0.1)$ & $0.1(0.1)$ & $12.6(2.1)$ & $2.6(1.2)$ & $0.0(0.1)$ & $10.0(1.4)$ & $<0.001$ \\
\hline
\end{tabular}

MSLT $=$ multiple sleep latency time.
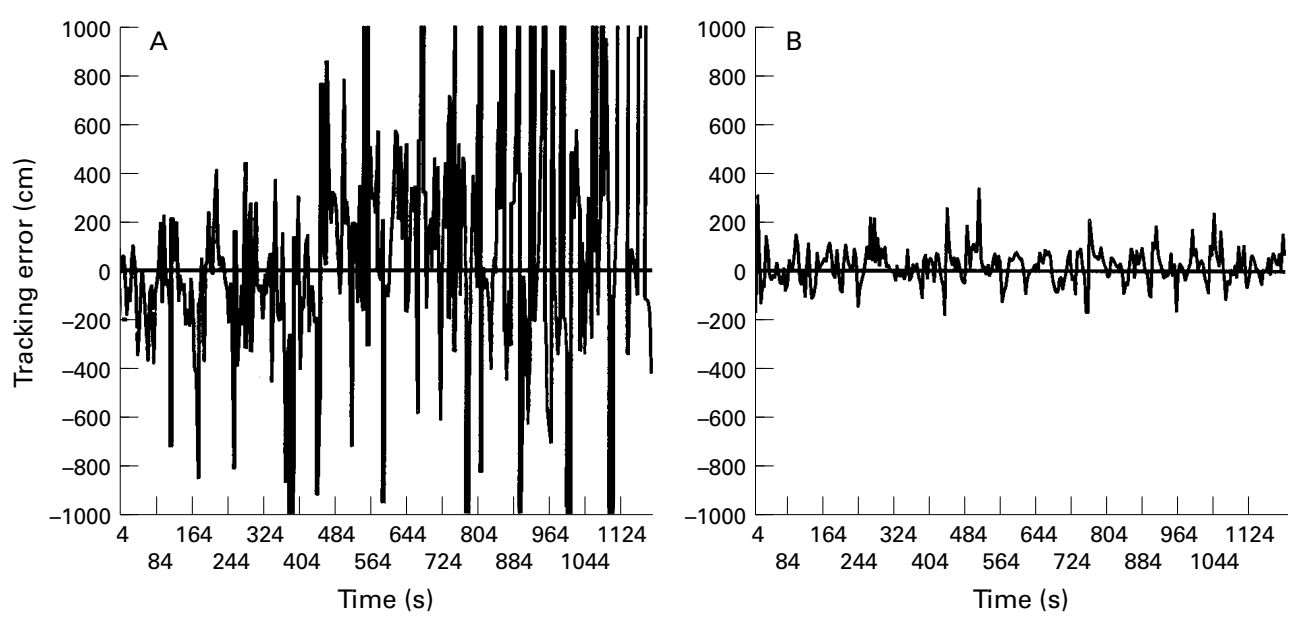

Figure 3 Tracking error for a 20 minute test run in a patient while (A) untreated and (B) after CPAP therapy. If the subject kept the cursor always centred in the box the tracking would be represented by a straight line. Deviation above and below the line indicates movement left and right of the centre of the screen. There was considerable improvement in tracking error in this individual with treatment.

missed responses (1.7 (95\% CI 0.9 to 2.3$)$ ), and number of times out of bounds $(10.0(95 \%$ CI 7.9 to 13.6)) but not for response time (0.1 $(95 \%$ CI -0.3 to 0.2$) \mathrm{s})$. Performance in the control subjects showed no significant testretest changes in primary or secondary outcome measures (table 2). Figure 3 shows the typical improvement in tracking error for a patient with OSA whose initial performance was impaired. Figure 4 shows the mean improvement for all patients and demonstrates that tracking error remained poor in only one patient with OSA.

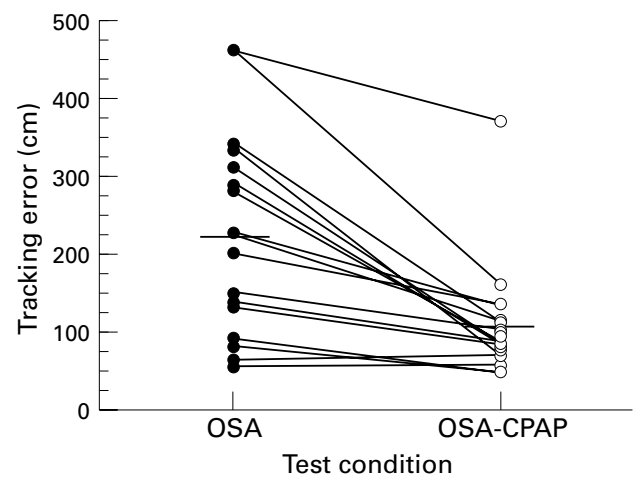

Figure 4 Mean values of all four runs for tracking error for individual patients with OSA (O) and after (O) treatment. Group means indicated by horizontal lines (difference, $p<0.001$ ). Only one subject did not improve to the control range.
SLEEPINESS

As expected, sleepiness as measured by MSLT was unchanged in controls (12.3 (6) versus 12.9 (5.9) $\min , \mathrm{p}=0.6$ ) but improved significantly in patients with CPAP treatment $(7.2$ (6) versus 13.2 (6.7) $\mathrm{min}, \mathrm{p}<0.001)$ There was no difference in MSLT between treated patients with OSA and controls.

\section{CORRELATIONS}

Change (improvement) in tracking error was highly correlated with improvement in sleepiness (as measured by MSLT) as seen in fig 5.

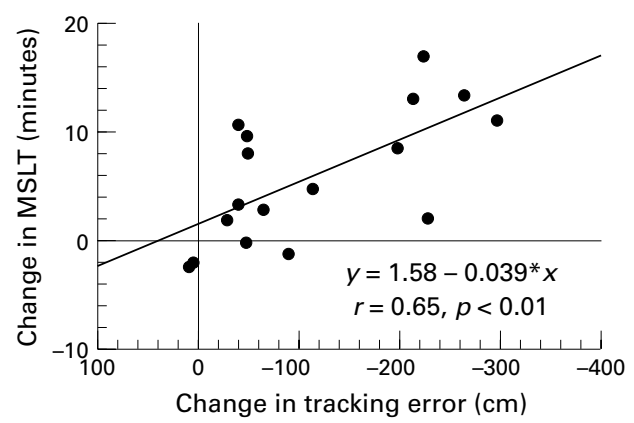

Figure 5 Change in tracking error versus a change in sleepiness (as measured by MSLT). The higher the change in MSLT (which indicates less sleepiness) the greater improvement in performance. 
Improvements in other outcome measures were not correlated with changes in sleepiness. While the change in MSLT accounts for $43 \%$ of the variance in tracking error improvement, factors other than sleepiness are obviously involved in determining driving performance.

\section{Discussion}

Treatment of apnoea with nasal CPAP resulted in significant improvement in simulated driving performance in this group of patients with OSA. Nocturnal sleep returned to normal and only one patient had any residual daytime complaints. Both primary (tracking) and secondary (response time, correct responses) performance measures improved significantly with treatment and only correct and missed responses, while improved, remained statistically different from controls.

With any performance test there is a learning curve and improvements between testings could be attributed to practice effects. To address this, practice runs were given to both groups at both points which, we believe, represents a particular strength of this study. Both controls and patients demonstrated a similar rate of learning when they were first tested. As there was no improvement in control subjects with repeat testing we feel that the improvement in performance can be attributed to the beneficial effect of nasal CPAP on sleep and breathing.

While all but one patient returned to normal performance, the time course of improvement cannot be determined in this study as subjects had repeated testing on only a single occasion and the time between tests was not the same for all subjects. Previous work by Lamphere and colleagues has shown that sleepiness improves within two weeks of treatment with CPAP. ${ }^{11}$ Cognitive function also improves with treatment $^{12}$ but may take longer. Although our patients were studied an average of nine months after starting treatment, two were retested after four weeks of CPAP treatment and their tracking error returned to the control range. The others who were tested later might also have improved within 2-4 weeks but further study with measurements at specified times after initiating CPAP treatment are necessary to answer this conclusively. In our patients the reported rate of compliance was high although objective measures of CPAP compliance are necessary to determine the time course and degree of improvement in performance.

One patient remained very poor in his divided attention performance. This patient was discovered to have significant underlying periodic limb movement disorder which disrupted his sleep and presumably produced daytime sleepiness. This was not recognised on his initial diagnostic study when sleep disruption due to severe apnoea obscured this finding. On his CPAP titration study there were periodic limb movements noted but they were not associated with arousal, probably because of the large amounts of rebound slow wave sleep from which arousal would have been blunted. This case highlights the need for follow up in patients with OSA once CPAP has been prescribed. Although sleep apnoea is one of the commonest causes of daytime sleepiness, there may be co-existing conditions which produce ongoing sleepiness and influence daytime function.

Previous studies have shown improvement in performance with CPAP. ${ }^{1314}$ Findley was the first to show improvement in performance on the computer based program Steer Clear. This task, initially described as a driving simulator, is really a choice reaction task requiring sustained vigilance. Both Findley ${ }^{13}$ and Engleman et al ${ }^{14}$ reported improvement in performance on this task with CPAP treatment. Haraldsson and colleagues, using an advanced car driving simulator, have reported both short and long term improvement in driving performance in patients treated with uvulopalatopharyngoplasty. ${ }^{15}$ Thus, improvement in performance in this study with CPAP treatment was not unexpected. Nonetheless it is reassuring to see improvement in virtually every patient in this study. However, application of these results to the sleep apnoea population at large is somewhat limited since all subjects in this study were male. No women were enrolled because, at the time when the initial study was started, ${ }^{6}$ the work of Young et al had not been published and the International Classification of Sleep Disorders ${ }^{9}$ listed a much higher male to female ratio for OSA.

Based on clinical history, many patients with OSA report no difficulties with their ability to drive. In this study there were patients whose pretreatment performance was not different from the control subjects. Others, however, were quite impaired. The clinical history may show differences in self-reported automobile accidents after CPAP treatment. Suratt and Findley found a fall in self-reported accidents or near accidents from $41 \%$ to $5 \%$ of a group of 22 patients treated with CPAP. ${ }^{16}$ In contrast, we recently reported marked differences (in the opposite direction) in self-reported driving difficulties between the initial clinic visit and follow up after treatment with CPAP in patients with OSA. ${ }^{17}$ Patients may be very reluctant to admit difficulties with driving because of the potential for loss of licence. Such discrepancies complicate the task of determining ability to operate a motor vehicle since most decisions are based on subjective data. Objective measures such as the DADT may become important additions to the process of determining ability to drive. Comparisons with subjects' driving records and/or on the road testing will be needed before this laboratory based test can be used in day to day clinical management. Such comparisons are now underway.

The authors wish to thank Sandra Halko and Lynne Babin for technical assistance and Darlene Taranik for secretarial expertise.

The Ontario Thoracic Society, Medical Research Council of Canada, and the Ontario Ministry of Transportation.

1 George CF, Nickerson P, Hanley P, Millar T, Kryger M. Sleep apnoea patients have more automobile accidents. Lancet 1987;i:447.

2 Findley LJ, Unverzagt ME, Suratt PM. Automobile accidents involving patients with obstructive sleep apnea. Am Rev Respir Dis 1988;138:337-40. 
3 Aldrich MS. Automobile accidents in patients with sleep disorders. Sleep 1989;12:487-94.

4 Haraldsson P, Carenfelt C, Diderichsen F, Nygren A, Tingvall C. Clinical symptoms of sleep apnea syndrome and automobile accidents. ORL 1990;52:57-62.

5 Strohl KP, Bonnie RJ, Findley L, Fletcher EC, Getsy J, Kryger M, et al. Sleep apnea, sleepiness and driving risk. Am $\mathcal{F}$ Respir Crit Care Med 1994;150:1463-73.

6 George CFP, Boudreau AC, Smiley A. Simulated driving performance in patients with obstructive

7 Respir Crit Care Med 1996;154:175-81.

7 Gould GA, Whyte KF, Rhind GB, Airlie MAA, Catterall JR, Shapiro CM, et al. The sleep hypopnea syndrome. Am Rev Respir Dis 1988;137:895-8.

8 Young T, Palta M, Dempsey J, Skatrud J, Weber S, Badr $\mathrm{S}$. The occurrence of sleep-disordered breathing among S. The occurrence of sleep-disordered breathing am

9 ICSD (International Classification of Sleep Disorders). Diagnostic and coding manual. Rochester, Minnesota: American Sleep Disorders Association, 1990.

10 Carskadon MA, Dement WC, Mitler MM, Roth T, Westbrook PR, Keenan S. Guidelines for the Multiple Sleep Latency Test (MSLT): a standard measure of sleepiness. Sleep 1986;9:519-24.
11 Lamphere J, Roehrs T, Wittig R, Zorick F, Conway WA, Roth T. Recovery of alertness after CPAP in apnoea. Chest 1989;96:1364-7.

12 Bearpark H, Grunstein R, Touyz S, Channon L, Sullivan C. Cognitive and psychological dysfunction in sleep apnea before and after treatment with CPAP. Sleep Res 1987;16: 303.

13 Findley L, Fabrizio M, Knight H, Norcross B, Leftward A, Suratt P. Driving simulator performance in patients with sleep apnea. Am Rev Respir Dis 1989;140:529-30.

14 Engleman HM, Martin SE, Deary IJ, Douglas NJ. Effect of continuous positive airway pressure treatment on daytime drome. Lancet 1994:343:572-5.

15 Haraldsson P-O, C Carenfelt, M Lysdahl, J Törnros. Long term effect of uvulopalatopharyngoplasty on driving performance. Arch Otolaryngol Head Neck Surg 1995;121: form

16 Suratt P, Findley L. Effect of nasal CPAP treatment on automobile driving simulator performance and on self reported automobile accidents in subjects with sleep apnea. Am Rev Respir Dis 1992;145:A169.

17 George CFP, Flaherty BA, Smiley A. Driving and sleep apnea: self reported accidents. Sleep Res 1995;24A:305. 